\title{
Prospective evaluation of fluorescence in situ hybridization for diagnosing urothelial carcinoma
}

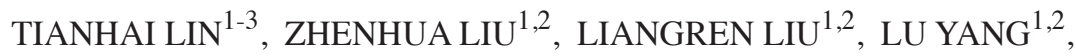 \\ PING HAN $^{1,2}$, PENG ZHANG ${ }^{1,2}$ and QIANG WEI ${ }^{1,2}$ \\ ${ }^{1}$ Department of Urology, ${ }^{2}$ Institute of Urology, West China Hospital, Sichuan University, Chengdu, \\ Sichuan 610041, P.R. China; ${ }^{3}$ Department of Translational Molecular Pathology, \\ University of Texas M.D. Cancer Center, Houston, TX 77030, USA
}

Received March 14, 2016; Accepted January 31, 2017

DOI: $10.3892 / 01.2017 .5926$

\begin{abstract}
Urothelial carcinoma (UC) is the most common type of cancer of the bladder and upper urinary tract, and is characterized by a high risk of recurrence and progression. Urine fluorescence in situ hybridization (FISH) is a technique that detects genetic aberrations in exfoliated cells in the urine, with specific probes for chromosomes 3, 7 and 17 and the p16 gene. To evaluate the diagnostic value of FISH in UC, 119 patients from November 2010 to June 2012 with suspected UC were recruited into a prospective, cross-sectional study and were followed up for 12-30 months. These patients received voided urine cytology and FISH tests, and underwent cystoscopy and/or ureteroscopy as a reference standard. The final diagnoses confirmed 73 patients with UC, located in the bladder, upper urinary tracts or the two. The sensitivity of FISH for detecting UC was superior to cytology, irrespective of tumor grade and stage: Overall, 80.8 vs. $32.9 \%(\mathrm{P}<0.001)$; low grade, 75.8 vs. $12.1 \%(\mathrm{P}<0.001)$; high grade, 85 vs. $50 \%$ $(\mathrm{P}<0.005)$; non-muscle-invasive, 81.1 vs. $28.3 \%(\mathrm{P}<0.001)$ and muscle-invasive, 80 vs. $45 \%(\mathrm{P}<0.05)$, respectively. The specificities of the two tests were similar; overall, the specificity was $89.1 \%$ for cytology vs. $100 \%$ for FISH, and no significant difference was observed between the methods. Notably, FISH exhibited $100 \%$ sensitivity for cytologically non-diagnostic UC, but $33.3 \%$ specificity. In conclusion, FISH is a reliable and non-invasive diagnostic tool for bladder and upper urinary tract UC, particularly in patients with low-grade or early stage tumors.
\end{abstract}

Correspondence to: Professor Qiang Wei, Department of Urology, West China Hospital, Sichuan University, 37 Guo Xue Xiang, Wuhou, Chengdu, Sichuan 610041, P.R. China

E-mail: weiqiang933@126.com

Key words: fluorescence in situ hybridization, transitional cell carcinoma, urothelial carcinoma, diagnosis, cytology, bladder cancer, sensitivity, specificity

\section{Introduction}

Urothelial carcinoma (UC) is the most common type of cancer of the bladder and upper urinary tract $(1,2)$. In the United States, there are 76,960 incident cases and 31,540 cancer-associated mortalities caused by bladder cancer each year (3). Furthermore, UC accounts for $\sim 80,500$ incident cases and 32,900 mortalities in China annually (4). With an annual incidence of $0.69-0.73 / 100,000$ per year, upper tract urothelial carcinoma (UTUC) is less common compared with bladder urothelial carcinoma (BUC) (5), but UTUC is often diagnosed concurrently with BUC. Although the majority of tumors are non-muscle-invasive when initially diagnosed, UC is characterized by a high risk of recurrence and progression. The 'Tumor, Node, Metastases' (TNM) classification system is widely used for staging UC (6). Of patients with the stage Ta-T2, $\sim 60 \%$ relapse within three years of initial treatment $(7,8)$, and multiple or recurrent lesions may indicate a lifelong risk of progression (9). UC is associated with a number of risk factors, with tobacco smoking considered to be the most important $(10,11)$. A previous study confirms that smoking exposure confers risk to the recurrence rate for smokers and non-smokers (12).

Diagnosis and surveillance of UC generally involve invasive procedures. Physical examination typically finds a limited number of clues, whilst in rare and advanced cases masses may be palpable. Cystoscopy, ureteroscopy and biopsy remain the standard diagnostic tools for UC. However, endoscopy is usually associated with complications including hemorrhage, infection and perforation, and has serious limitations in certain patients with anatomical abnormalities or history of urinary tract reconstruction, for example urinary diversion or ureteroileal anastomosis (13).

Urine-based diagnostic tests are non-invasive and convenient, but inaccurate. Urine cytology for exfoliated cells exhibits excellent specificity, but the overall sensitivity is low, particularly for low-grade tumors, ranging between $4-31 \%(13,14)$. Numerous biomarkers for tumor-associated proteins including human complement factor $\mathrm{H}$-related protein and nuclear matrix protein 22 , and the diagnostic test ImmunoCyt have been introduced to achieve a higher sensitivity. However, as these tests are immunology-based approaches, 
these markers are susceptible to urinary tract infections and intravesical therapies, resulting in a trade-off between sensitivity and specificity (15).

Fluorescence in situ hybridization (FISH) is a technique that allows the visualization of genetic aberrations in many types of tumor. In UC, the most prominent types of genetic changes are the loss of the p16 gene at 9p21 and aneuploidies of chromosomes 3, 7 and 17. Therefore, multitargeted multicolor FISH probes that are specific for these aberrations have been developed and tested for the diagnosis of UC (16).

Over the previous decade, several studies have evaluated the diagnostic value of FISH in UC, but the clinical value and cost-efficiency remains incompletely characterized $(17,18)$. As important data has arisen from case-control designs (19-21) or focused on BUC or UTUC exclusively (22-25), the present study was designed to assess the diagnostic value of FISH for detecting UC prospectively, and to evaluate the utility of FISH in association with cytology as a non-invasive diagnostic technique.

\section{Patients and methods}

Between November 2010 and June 2012, patients with suspected UC in West China Hospital, Sichuan University (Chengdu, China) were enrolled consecutively in a prospective, cross-sectional study. The majority of patients presented with gross hematuria, unexplained hydroureterosis or hydronephrosis or abnormal imaging findings in the urinary tract, for example; masses or filling defects.

Once recruited, patient samples underwent urine cytology and FISH assays, followed by cystoscopy and/or ureteroscopy within one week of urine collection. In patients with negative cystoscopy but either positive cytology or FISH, ureteroscopy was mandatory. If indicated, a biopsy or resection was performed. Patients negative for endoscopy in the initial assessment underwent a follow-up endoscopy every 3-6 months. The diagnostic procedures are illustrated in Fig. 1. The present study was approved by the West China Hospital (Chengdu, China) Ethics Committee and written informed consent was obtained from each patient.

Prior to urine collection, each patient was instructed to empty the bladder completely, then drink $500 \mathrm{ml}$ water. After 1-2 h, two urine specimens of voided midstream urine were collected, $\geq 100 \mathrm{ml}$ per sample, and analyzed within one hour.

For cytology, the urine specimens were centrifuged at $600 \times \mathrm{g}$ for $10 \mathrm{~min}$ at room temperature, and the sediment fractions were sectioned for Papanicolaou staining and cytological diagnosis. Atypical or equivocate interpretations were reported as non-diagnostic.

The urine FISH assays were conducted with multitargeted, multicolor and commercially available kits (Bladder Cancer kits, Beijing GP Medical Technologies, Ltd., Beijing, China) according to the protocol of the manufacturer. The slides were prepared using the same procedure as the cytological analysis, and were denaturized and hybridized with two pairs of probes: Chromosome enumeration probe (CEP) 3/7 for chromosomes 3 and 7, and glucagon-like receptor cyclin-dependent kinase inhibitor 2A (p16)/CEP17 for the locus 9p21 and chromosome 17 , respectively. Subsequent to washing of the slides, the nuclei were counterstained with 4,6-diamidino-2-phenylindole and were analyzed using a fluorescence microscope. A total of 100 unoverlapped cells were evaluated consecutively. An analyzable reading required a minimum $80 \%$ hybridization rate.

One key step was to determine the cutoff for a positive reading. The fluorescent signals in fresh voided urine specimens collected from 20 healthy control individuals in West China Hospital were analyzed, and the cutoff was determined as the mean percentage in healthy controls $+3 \mathrm{x}$ standard deviation. For polysomy of CEP3, CEP7 and CEP17, the cutoff was 7,8 and $8 \%$ respectively. For p16, $>10 \%$ of the counted nuclei with either homozygous or hemizygous loss were considered abnormal. A positive FISH result was given if any single cutoff was exceeded.

To determine the sensitivity and specificity profiles, endoscopy and subsequent histological diagnosis were used as the reference standard. The biopsy was performed by transurethral resection or open surgery, and the tissues were sectioned by routine procedures. The tumor was staged and graded in accordance with the TNM classification (6) and the World Health Organization 2004 classification (26).

All tests were examined by two pathologists independently. The consensus was made and reported, and a third senior pathologist was consulted if required. All reviewers were blinded to the results of other tests and the clinical records except for suspicion of UC.

In order to define the difference in the diagnostic values between FISH and cytology in a sufficient number of patients, a binomial McNemar test for paired data and $\chi^{2}$ test for unpaired data were used. The correct sample size was estimated following a method for paired diagnostic tests $(27,28)$. The probable difference in the sensitivity and the specificity between the two assays was assumed based on a previous meta-analysis (14), and an $80 \%$ power was chosen to identify the disagreement. $\mathrm{P}<0.05$ was considered to indicate a statistically significant difference. All statistical analyses were performed using IBM SPSS Statistics version 23.0.

\section{Results}

Demographic and clinical characteristics of the patients. To compare the diagnostic value between FISH and cytology, a minimum of 65 patients were required and the present study recruited 123. A total of 119 patients qualified for the final analysis, and 4 were excluded due to loss to follow-up.

The demographic and clinical characteristics of the qualified patients are listed in Table I. A total of 16 patients exhibited histologically confirmed BUC with at least one transurethral resection prior to enrollment. A total of $13 \mathrm{had}$ been diagnosed with other urinary disorders, including benign prostate hyperplasia and renal cysts. Only 1 patient exhibited a previous lateral nephrectomy due to renal tuberculosis. Gross hematuria was exhibited in 103 patients, and the most common findings on imaging were masses, followed by filling defects in the upper urinary tracts. As $33.6 \%$ of the patients in the present study were associated with a current or prior smoking history, the FISH results, listed by smoking status in Table II, demonstrate that tobacco smoking contributed significantly to UC prevalence $(\mathrm{P}<0.05)$. However, the correlation between smoking status and FISH sensitivity was not established in the present study $(\mathrm{P}>0.05)$. 
Table I. Demographic and clinical characteristics of 119 patients with suspected urothelial carcinoma.

\begin{tabular}{lc}
\hline Characteristic & Value \\
\hline Age, years & \\
Mean \pm standard deviation & $60.4 \pm 17$ \\
Range & $25-89$ \\
Female sex, n (\%) & $31(26.1)$ \\
Current or prior tobacco smoking, $\mathrm{n}(\%)$ & $40(33.6)$ \\
History of urinary disorders, $\mathrm{n}(\%)$ & \\
Urothelial carcinoma & $16(13.4)$ \\
Benign disorders & $13(10.9)$ \\
Gross hematuria, $\mathrm{n}(\%)$ & $103(86.6)$ \\
Positive findings in ultrasound, & $76(63.9)$ \\
IVU, CTU or MRU imaging, $\mathrm{n}(\%)$ & \\
\hline
\end{tabular}

IVU, intravenous urography; CTU, computed tomographic urography; MRU, magnetic resonance urography.

The final histological diagnoses of the 73 confirmed UC are summarized in Table III. A total of 5 cases of renal cell carcinoma were identified, and the remaining 41 patients were tumor-free throughout the present study. The patients were followed up for an average period of 18.7 months, ranging from 12-30.

Diagnostic values of cytology and FISH. To compare the performance of cytology, FISH and the two as simultaneous tests for diagnosing UC, the sensitivities, specificities and predictive values were calculated and are listed in Table IV. Urine cytology was positive for malignancy in 24 of 73 patients and negative in 49. A total of 9 samples were non-diagnostic, due to atypical or equivocal interpretations, and were considered negative in the analysis. FISH was positive in 59 and negative in 14 UC cases. A total of 4 samples that lacked sufficient cells were considered negative, and comprised 2 patients with BUC, 1 patient with kidney cancer, and 1 true negative. Additional analysis of the benefit of combining cytology and FISH as a simultaneous test revealed that the increase in sensitivity and negative predictive value (NPV) was $1.4 \%$. In contrast, combining the tests decreased the specificity and positive predictive value (PPV) by 10.9 and 7.7\%, respectively.

As the major criticism on urine FISH and cytology is the lack of sensitivity for low-grade and early stage UC, the data were examined by tumor grade, stage and location. As demonstrated in Table V, cytology was considerably less effective for highly differentiated or non-muscle-invasive tumors. Out of 33 low-grade UC, only 4 were identified by cytology. Conversely, the sensitivity of FISH was stable for tumor grade and stage.

Compared with cytology, FISH exhibited higher sensitivity for detecting UC, as illustrated in Fig. 2. The discrepancy was particularly conspicuous between low-grade and early stage UC, as the sensitivity for low grade FISH was $75.8 \%$ vs. $12.1 \%$ for low grade cytology, and the sensitivity for early stage FISH was $81.1 \%$ vs. $28.3 \%$ for early stage cytology, respectively (FISH vs. cytology, $\mathrm{P}<0.001$ ). In 19 patients with UTUC

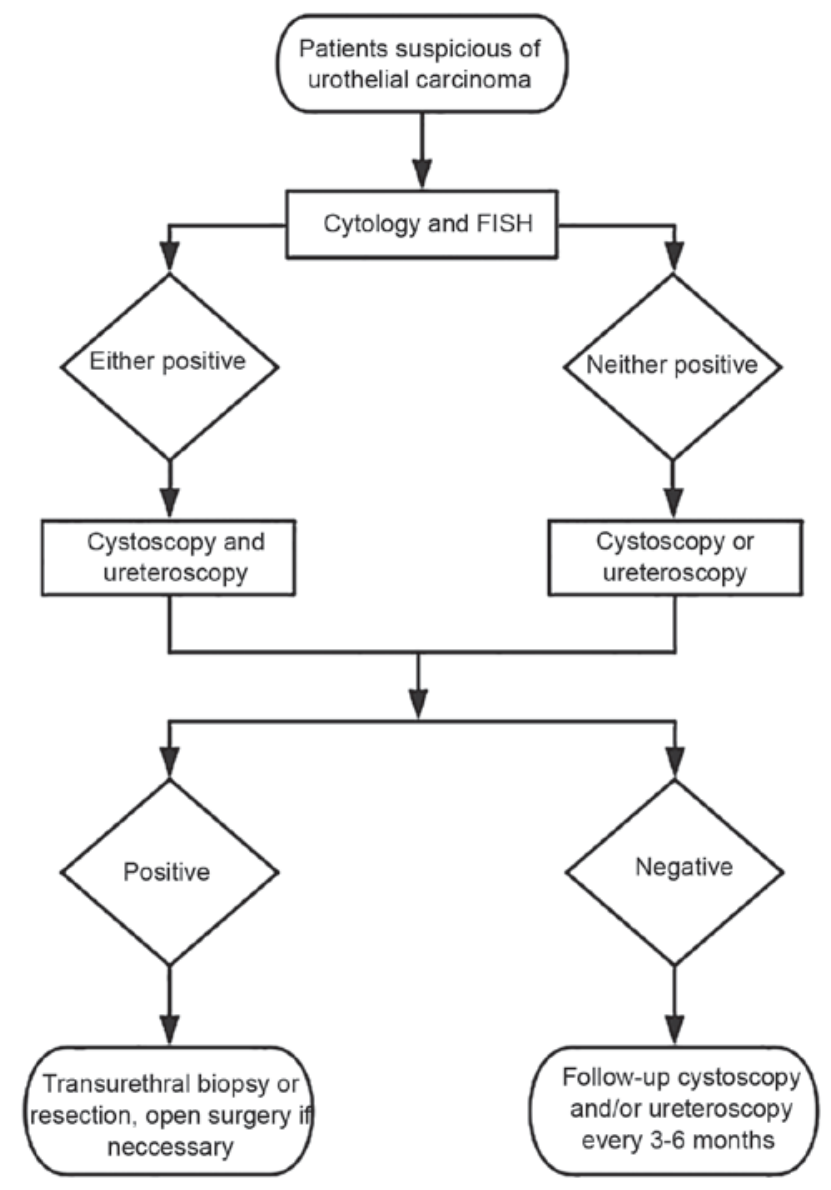

Figure 1. Flow diagram demonstrating the diagnostic procedures in the present study. FISH, fluorescence in situ hybridization.

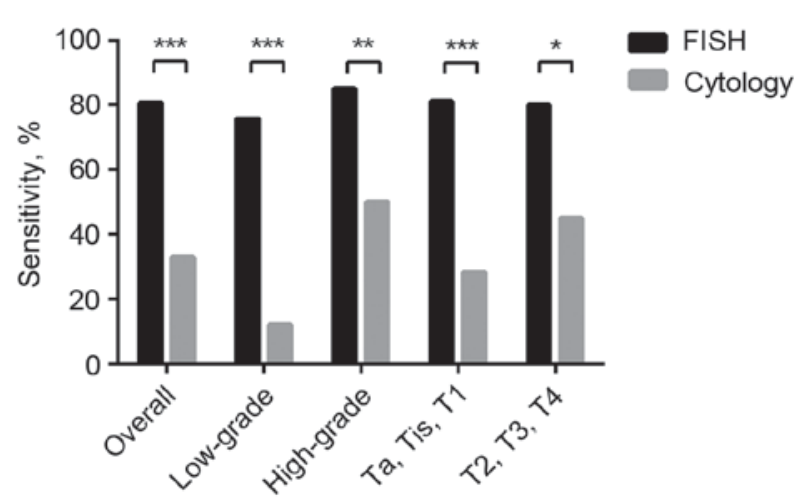

Figure 2. Comparison of the sensitivities of FISH and cytology for diagnosing urothelial carcinoma. ${ }^{*} \mathrm{P}<0.05,{ }^{* *} \mathrm{P}<0.005,{ }^{* * * *} \mathrm{P}<0.001$ between FISH and cytology. FISH, fluorescence in situ hybridization; Ta, non-invasive papillary carcinoma; Tis, carcinoma in situ; $\mathrm{T} 1$, tumor invades subepithelial connective tissue; T2, Tumor invades muscle; T3, tumor invades beyond muscle; $\mathrm{T} 4$, tumor invades adjacent organs or fat.

without concomitant BUC, FISH exhibited significantly higher sensitivity compared with cytology $(\mathrm{P}<0.01)$. When determining specificity, 5 patients with negative histology were diagnosed with UC by FISH, whereas cytology exhibited no false positives. However, the difference was not significant $(\mathrm{P}=0.063)$.

Additionally, of the 9 cytologically non-diagnostic patients, 8 were positive using FISH, including all 6 patients with UC 
Table III. Tumor characteristics of 73 patients diagnosed with urothelial carcinoma by histological diagnosis.

\begin{tabular}{lc}
\hline Characteristic & Value, $\mathrm{n}(\%)$ \\
\hline Location of lesion & \\
Urinary bladder & $52(71.2)$ \\
Upper urinary tract & $19(26)$ \\
Both & $2(2.7)$ \\
Tumor grade & \\
Low & $33(45.2)$ \\
High & $40(54.8)$ \\
Tumor stage & \\
pTa & $30(41.1)$ \\
pTis & $1(1.4)$ \\
pT1 & $22(30.1)$ \\
pT2 & $9(12.3)$ \\
pT3 & $6(8.2)$ \\
pT4 & $5(6.8)$
\end{tabular}

pTa, non-invasive papillary carcinoma; pTis, carcinoma in situ; pT1, tumor invades subepithelial connective tissue; pT2, tumor invades muscle; pT3, tumor invades beyond muscle; pT4, tumor invades adjacent organs or fat.

in this group. There was 1 false positive patient who exhibited a history of UC prior to the present study, and the other was diagnosed with kidney cancer. The sensitivity and specificity of FISH when used to diagnose cytologically equivocal samples were 100 and $33.3 \%$ respectively.

\section{Discussion}

The purpose of the present study was to evaluate the diagnostic value of FISH for diagnosing UC, and to assess the clinical consequences of FISH-based diagnostics in association with cytology. In a cohort of patients with suspected UC, it was revealed that FISH exhibited a higher sensitivity, PPV and NPV compared with cytology across tumor grade, stage and location. Cytology is associated with an excellent specificity, but the sensitivity is greatly reduced in low-grade UC. The utility of the two methods as a simultaneous test was investigated, but the data suggest that the combination did not improve the accuracy of FISH.

FISH is effective, but not always efficient, in the diagnosis of UC. The advantage of FISH in sensitivity over cytology is reduced subsequent to the exclusion of superficial cancer from the analysis, indicating that tumor grade and stage may affect the accuracy (29). Although tobacco smoking is suggested to affect the wider prevalence of UC, and there is a higher detection rate in heavy smokers (30), the present study was only able to validate the former.

The data of the present study suggest that the high sensitivity of FISH across UC stage and grade is not coincidental (31). Potential explanations for the discrepancy between the results of the present study and other observations may be attributed to selection and observer bias. However, the approaches to the 
Table IV. Sensitivity, specificity and predictive value of cytology, FISH and both as a simultaneous test for diagnosis of UC in 119 patients.

\begin{tabular}{|c|c|c|c|c|c|c|c|}
\hline &,$+ \mathrm{n}$ &,$- \mathrm{n}$ & Total, $\mathrm{n}$ & Sen $\%$ & Spe $\%$ & PPV \% & NPV \% \\
\hline Cytology & & & & 32.9 & 100 & 100 & 48.4 \\
\hline+ & 24 & 0 & 24 & & & & \\
\hline- & 49 & 46 & 95 & & & & \\
\hline FISH & & & & 80.8 & 89.1 & 92.2 & 74.5 \\
\hline+ & 59 & 5 & 64 & & & & \\
\hline- & 14 & 41 & 55 & & & & \\
\hline Simultaneous & & & & 82.2 & 89.1 & 92.3 & 75.9 \\
\hline+ & 60 & 5 & 65 & & & & \\
\hline- & 13 & 41 & 54 & & & & \\
\hline Total & 73 & 46 & 119 & & & & \\
\hline
\end{tabular}

UC, urothelial carcinoma; FISH, fluorescence in situ hybridization; Sen, sensitivity; Spe, specificity; PPV, positive predictive value; NPV, negative predictive value.

Table V. Sensitivity of FISH and cytology for 73 urothelial carcinoma by tumor grade, stage and location.

\begin{tabular}{lccc}
\hline Characteristic & $\mathrm{n}$ & $\begin{array}{c}\text { Cytology } \\
\text { sensitivity, } \%\end{array}$ & $\begin{array}{c}\text { FISH } \\
\text { sensitivity, \% }\end{array}$ \\
\hline Grade & & & \\
Low & 33 & 12.1 & 75.8 \\
High & 40 & 50 & 85 \\
Stage & & & \\
Ta, Tis, T1 & 53 & 28.3 & 81.1 \\
T2, T3, T4 & 20 & 45 & 80 \\
Location & & & \\
Bladder & 52 & 32.7 & 84.6 \\
Upper urinary tract & 19 & 31.6 & 73.7 \\
Both & 2 & 50 & 100 \\
\hline
\end{tabular}

Ta, non-invasive papillary carcinoma; Tis, carcinoma in situ; T1, tumor invades subepithelial connective tissue; T2, tumor invades muscle; T3, tumor invades beyond muscle; T4, tumor invades adjacent organs or fat.

urine samples of the present study should also be considered. Firstly, only fresh voided urine specimens were examined. In addition, the pathology laboratory performing the tests was in the vicinity of the outpatient building and urology unit of the West China Hospital (Chengdu, China), allowing the specimens to be processed and prepared within short time periods, to reduce degeneration of the samples as much as possible. All FISH assays were performed on independent samples rather than the remains or the same slides from cytology to minimize the interference from previous manipulations. Lastly, the hybridization rate was considered a key indicator of the quality control, for example; samples that failed to reach an $80 \%$ hybridization rate were retested until the criteria were met. As only $4 / 119,3.4 \%$, FISH results were deemed 'uninformative', careful handling and strict standard of quality control may improve the sensitivity of FISH assay for obscure abnormalities in urine specimens. Another explanation for the observed discrepancy between the present study and previous studies may be the heterogeneity in the diagnostic criteria across different studies.

As the first commercially available FISH kit for diagnosing bladder cancer, UroVysion Bladder Cancer Kit (Abbott Molecular, Inc., USA), was approved by the United States Food and Drug Administration in 2001, the UroVysion criteria has been widely used in clinical practice and studies, with continuous adaptions. Bubendorf et al (32) suggested that the detection of $\leq 4$ tetrasomic cells in each sample should be considered normal in order to improve diagnostic value. Concurrently, Reynolds et al (33) demonstrated that FISH had improved sensitivity vs. cytology, but decreased specificity due to a greater number of false-positive results. Considering the lack of validation of the UroVysion criteria in the Chinese population, the threshold was determined by performing FISH assays on samples from healthy donors. Additionally, Huang et al (22) reported similar results in BUC with this approach, and a direct comparison with the UroVysion criteria established by Cui et al (34) demonstrated that the donor-derived criteria is more effective.

From the literature, FISH possesses a substantially higher sensitivity for UTUC $(25,35-37)$, whereas cytology is only positive in $20 \%$ patients without concurrent bladder UC, including invasive or high-grade UTUC $(38,39)$. As UTUC shares identical karyotypic profiles with BUC (40), it is hypothesized that FISH serves an effective role for detecting UTUC. However, the generally small sample size in previous studies, owing to the low incidence of UTUC, undermines their strength of evidence.

Another application of FISH is to supplement cytological analysis. Previous studies have demonstrated that FISH possesses a remarkably high accuracy in patients with suspicious, atypical or negative cytology, and the potential to predict UC development-patients with atypical cytology and false positive FISH at the initial assessment commonly develop UC 
within $15-22$ months $(41,42)$, and a preceding positive FISH result is associated with tumor relapse in $86 \%$ of UC surveillance cases, including all high-grade recurrences (43). These data suggest that 'false positive' FISH reports should not be ignored, as they may indicate underlying tumorigenesis.

However, the FISH assay possesses limitations. This approach is more complicated and expensive compared with cytology, requiring a fluorescence microscope with commercial kits and specially trained laboratory staffs to visualize and interpret the results. Considering these resources, performing FISH assays subsequent to positive cytology results is not realistic, as positive cytology results are reliable due to the high specificity of cytology for UC. FISH is also not cost-effective when low incidences of UC are expected, due to the reduced chance of diagnosing tumors. In particular, the performance of FISH is suboptimal when used as a reflex test concomitantly with cytology and UC screening in high risk populations $(24,44)$.

Given these arguments, refinements are required to maximize the potential of urine FISH assays. Bladder and upper urinary tract washing may increase the probability of the detection of UC, as low-grade tumors are prone to shed fewer neoplastic cells into the urinary tract compared with advanced tumors, thus remaining undetected by urine-based tests (45). Adequate training and calibrated protocol for the performance of urine FISH assays may reduce the misinterpretation caused by sample handling and improper criteria.

Notably, the present study is based on a cohort from a single institution, which may potentially suffer from limited patient numbers and selection bias. Notwithstanding the limitations, these data support the importance of urine FISH assays for diagnosing bladder and upper urinary tract UC. They also underscore the value of these assays in patients with low-grade and early stage tumors, in which cytology-based methods are unreliable.

\section{Acknowledgements}

The present study was supported/partially supported by Clinical Application Fund for Molecular Biology of the Chinese Medical Association (grant no. CAMB032010) and China Scholarship Council. The authors thank our colleagues from Department of Pathology who provided insight and expertise that greatly assisted the study.

\section{References}

1. Babjuk M, Oosterlinck W, Sylvester R, Kaasinen E, Böhle A, Palou-Redorta J and Rouprêt M; European Association of Urology (EAU): EAU guidelines on non-muscle-invasive urothelial carcinoma of the bladder, the 2011 update. Eur Urol 59: 997-1008, 2011.

2. Ries LAG, Young JL, Keel GE, Eisner MP, Lin YD and Horner MJ, (eds): SEER survival monograph: Cancer survival among adults: U.S. seer program, 1988-2001, patient and tumor characteristics. National Cancer Institute, SEER Program, NIH Pub. No. 07-6215, Bethesda, MD, 2007.

3. Siegel RL, Miller KD and Jemal A: Cancer statistics, 2016. CA Cancer J Clin 66: 7-30, 2016.

4. Chen W, Zheng R, Baade PD, Zhang S, Zeng H, Bray F, Jemal A, Yu QX and Jie H: Cancer statistics in China, 2015. CA Cancer J Clin 66: 115-132, 2016.

5. Munoz JJ and Ellison LM: Upper tract urothelial neoplasms: Incidence and survival during the last 2 decades. J Urol 164: $1523-1525,2000$.
6. Sobin LH, Gospodariwicz MK and Wittekind C, (eds): TNM classification of malignant tumours. UICC International Union Against Cancer. 7th ed: 262-265, 2009.

7. Lutzeyer W, Rübben $\mathrm{H}$ and Dahm H: Prognostic parameters in superficial bladder cancer: An analysis of 315 cases. J Urol 127: 250-252, 1982.

8. Heney NM, Ahmed S, Flanagan MJ, Frable W, Corder MP, Hafermann MD and Hawkins IR: Superficial bladder cancer: Progression and recurrence. J Urol 130: 1083-1086, 1983.

9. Herr HW: Natural history of superficial bladder tumors: 10- to 20-year follow-up of treated patients. World J Urol 15: 84-88, 1997.

10. Freedman ND, Silverman DT, Hollenbeck AR, Schatzkin A and Abnet CC: Association between smoking and risk of bladder cancer among men and women. JAMA 306: 737-745, 2011.

11. Zeegers MP, Tan FE, Dorant E and van Den Brandt PA: The impact of characteristics of cigarette smoking on urinary tract cancer risk: A meta-analysis of epidemiologic studies. Cancer 89: 630-639, 2000.

12. Crivelli JJ, Xylinas E, Kluth LA, Rieken M, Rink M and Shariat SF: Effect of smoking on outcomes of urothelial carcinoma: A systematic review of the literature. Eur Urol 65: 742-754, 2014.

13. Sanderson KM and Rouprêt M: Upper urinary tract tumour after radical cystectomy for transitional cell carcinoma of the bladder: An update on the risk factors, surveillance regimens and treatments. BJU Int 100: 11-16, 2007.

14. Hajdinjak T: Urovysion FISH test for detecting urothelial cancers: Meta-analysis of diagnostic accuracy and comparison with urinary cytology testing. Urol Oncol 26: 646-651, 2008.

15. Lotan Y and Roehrborn CG: Sensitivity and specificity of commonly available bladder tumor markers versus cytology: Results of a comprehensive literature review and meta-analyses. Urology 61: 109-118, 2003.

16. Sokolova IA, Halling KC, Jenkins RB, Burkhardt HM, Meyer RG, Seelig SA and King W: The development of a multitarget, multicolor fluorescence in situ hybridization assay for the detection of urothelial carcinoma in urine. J Mol Diagn 2: 116-123, 2000.

17. Bubendorf L: Multiprobe fluorescence in situ hybridization (UroVysion) for the detection of urothelial carcinoma-FISHing for the right catch. Acta Cytol 55: 113-119, 2011.

18. Mowatt G, Zhu S, Kilonzo M, Boachie C, Fraser C, Griffiths TR, N'Dow J, Nabi G, Cook J and Vale L: Systematic review of the clinical effectiveness and cost-effectiveness of photodynamic diagnosis and urine biomarkers (FISH, ImmunoCyt, NMP22) and cytology for the detection and follow-up of bladder cancer. Health Technol Assess 14: 1-331, iii-iv, 2010.

19. Kang JU, Koo SH, Jeong TE, Kwon KC, Park JW and Jeon CH: Multitarget fluorescence in situ hybridization and melanoma antigen genes analysis in primary bladder carcinoma. Cancer Genet Cytogenet 164: 32-38, 2006.

20. Marín-Aguilera M, Mengual L, Ribal MJ, Burset M, Arce Y, Ars E, Oliver A, Villavicencio H, Algaba F and Alcaraz A: Utility of a multiprobe fluorescence in situ hybridization assay in the detection of superficial urothelial bladder cancer. Cancer Genet Cytogenet 173: 131-135, 2007.

21. Dimashkieh H, Wolff DJ, Smith TM, Houser PM, Nietert PJ and Yang J: Evaluation of urovysion and cytology for bladder cancer detection: A study of 1835 paired urine samples with clinical and histologic correlation. Cancer Cytopathol 121: 591-597, 2013.

22. Huang JW, Mu JG, Li YW, Gan XG, Song LJ, Gu BJ, Fu Q, Xu YM and An RH: The utility of fluorescence in situ hybridization for diagnosis and surveillance of bladder urothelial carcinoma. Urol J 11: 1974-1979, 2014.

23. Li HX, Wang MR, Zhao H, Cao J, Li CL and Pan QJ: Comparison of fluorescence in situ hybridization, NMP22 bladderchek and urinary liquid-based cytology in the detection of bladder urothelial carcinoma. Diagn Cytopathol 41: 852-857, 2013.

24. Banek S, Schwentner C, Täger D, Pesch B, Nasterlack M, Leng G, Gawrych K, Bonberg N, Johnen G, Kluckert M, et al: Prospective evaluation of fluorescence-in situ-hybridization to detect bladder cancer: Results from the uroscreen-study. Urol Oncol 31: 1656-1662, 2013.

25. Huang WT, Li LY, Pang J, Ruan XX, Sun QP, Yang WJ and Gao X: Fluorescence in situ hybridization assay detects upper urinary tract transitional cell carcinoma in patients with asymptomatic hematuria and negative urine cytology. Neoplasma 59: 355-360, 2012.

26. Montironi R and Lopez-Beltran A: The 2004 WHO classification of bladder tumors: A summary and commentary. Int J Surg Pathol 13: 143-153, 2005. 
27. Connor RJ: Sample size for testing differences in proportions for the paired-sample design. Biometrics 43: 207-211, 1987.

28. Beam CA: Strategies for improving power in diagnostic radiology research. AJR Am J Roentgenol 159: 631-637, 1992.

29. Zhou AG, Hutchinson LM and Cosar EF: Urine cytopathology and ancillary methods. Surg Pathol Clin 7: 77-88, 2014

30. Sarosdy MF, Kahn PR, Ziffer MD, Love WR, Barkin J, Abara EO, Jansz K, Bridge JA, Johansson SL, Persons DL and Gibson JS: Use of a multitarget fluorescence in situ hybridization assay to diagnose bladder cancer in patients with hematuria. J Urol 176: 44-47, 2006.

31. Chen N, Gong J, Zeng H, Wei Q, Zhu YC, Chen M and Zhou Q: Value of fluorescence in situ hybridization of urine exfoliative cells in diagnosis of urinary bladder neoplasms. Sichuan Da Xue Xue Bao Yi Xue Ban 42: 109-113, 2011 (In Chinese).

32. Bubendorf L, Grilli B, Sauter G, Mihatsch MJ, Gasser TC and Dalquen P: Multiprobe FISH for enhanced detection of bladder cancer in voided urine specimens and bladder washings. Am J Clin Pathol 116: 79-86, 2001

33. Reynolds JP, Voss JS, Kipp BR, Karnes RJ, Nassar A, Clayton AC, Henry MR, Sebo TJ, Zhang J and Halling KC: Comparison of urine cytology and fluorescence in situ hybridization in upper urothelial tract samples. Cancer Cytopathol 122: 459-467, 2014.

34. Cui X, Jiang Y, Luo Y, Zhao J and Zhao L: The impact of various standards in the diagnosis of bladder cancer by fluorescence in situ hybridization. J Clin Urology 24: 889-891, 894, 2009.

35. Marin-Aguilera M, Mengual L, Ribal MJ, Musquera M, Ars E, Villavicencio $\mathrm{H}$, Algaba $\mathrm{F}$ and Alcaraz A: Utility of fluorescence in situ hybridization as a non-invasive technique in the diagnosis of upper urinary tract urothelial carcinoma. Eur Urol 51 409-415, 2007

36. Chen AA and Grasso M: Is there a role for FISH in the management and surveillance of patients with upper tract transitional-cell carcinoma? J Endourol 22: 1371-1374, 2008

37. Mian C, Mazzoleni G, Vikoler S, Martini T, Knüchel-Clark R, Zaak D, Lazica A, Roth S, Mian M and Pycha A: Fluorescence in situ hybridisation in the diagnosis of upper urinary tract tumours. Eur Urol 58: 288-292, 2010.
38. Smith AK, Larson BT, Berger A and Jones SJ: Is there a role for cytology in the diagnosis of upper tract urothelial cancer? J Urology 181: 132, 2009.

39. Messer J, Shariat SF, Brien JC, Herman MP, Ng CK, Scherr DS, Scoll B, Uzzo RG, Wille M, Eggener SE, et al: Urinary cytology has a poor performance for predicting invasive or high-grade upper-tract urothelial carcinoma. BJU Int 108: 701-705, 2011

40. Fadl-Elmula I: Chromosomal changes in uroepithelial carcinomas. Cell Chromosome 4: 1, 2005.

41. Skacel M, Fahmy M, Brainard JA, Pettay JD, Biscotti CV, Liou LS, Procop GW, Jones JS, Ulchaker J, Zippe CD and Tubbs RR: Multitarget fluorescence in situ hybridization assay detects transitional cell carcinoma in the majority of patients with bladder cancer and atypical or negative urine cytology. J Urol 169: 2101-2105, 2003.

42. Daniely M, Rona R, Kaplan T, Olsfanger S, Elboim L, Freiberger A, Lew S and Leibovitch I: Combined morphologic and fluorescence in situ hybridization analysis of voided urine samples for the detection and follow-up of bladder cancer in patients with benign urine cytology. Cancer 111: 517-524, 2007.

43. Gofrit ON, Zorn KC, Silvestre J, Shalhav AL, Zagaja GP Msezane LP and Steinberg GD: The predictive value of multi-targeted fluorescent in-situ hybridization in patients with history of bladder cancer. Urol Oncol 26: 246-249, 2008.

44. Ferra S, Denley R, Herr H, Dalbagni G, Jhanwar S and Lin O: Reflex urovysion testing in suspicious urine cytology cases. Cancer 117: 7-14, 2009.

45. Yoder BJ, Skacel M, Hedgepeth R, Babineau D, Ulchaker JC, Liou LS, Brainard JA, Biscotti CV, Jones JS and Tubbs RR: Reflex urovysion testing of bladder cancer surveillance patients with equivocal or negative urine cytology: A prospective study with focus on the natural history of anticipatory positive findings. Am J Clin Pathol 127: 295-301, 2007. 